\title{
Spectroscopy on the Wing: Investigating possible differences in protein secondary structures in feather shafts of birds using Raman spectroscopy
}

Christian M. Laurent*1,2,3; John M. Dyke*4; Richard B. Cook ${ }^{5}$; Gareth Dyke ${ }^{3}$ \& Roeland de $\mathrm{Kat}^{1,6}$

\author{
${ }^{1}$ Aerodynamics and Flight Mechanics, University of Southampton, UK, SO17 1BJ \\ ${ }^{2}$ Ocean and Earth Science, National Oceanography Centre, UK. SO17 1BJ \\ ${ }^{3}$ Department of Biology and Geology, Babes-Bolyai University, Romania. \\ ${ }^{4}$ School of Chemistry, University of Southampton, UK, SO17 1BJ \\ ${ }^{5}$ nCATS National Centre for Advanced Tribology Southampton, University of Southampton, \\ UK, SO17 1BJ \\ ${ }^{6}$ Faculty of Military Sciences, Netherlands Defence Academy, The Netherlands
}

*Corresponding Authors: jmdyke@soton.ac.uk

\section{christian_laurent@live.com}

Keywords:

Feathers, Raman Spectroscopy, Protein secondary structure, Feather keratin, Feather shaft,

\section{Abstract}

The central shaft of a bird's flight feather bears most of the aerodynamic load during flight and exhibits some remarkable mechanical properties. The shaft comprises two parts, the calamus and the rachis. The calamus is at the base of the shaft, while the rachis is the longer upper part which supports the vanes. The shaft is composed of a fibrous outer cortex, and an inner foam-like core. Recent nanoindentation experiments have indicated that reduced modulus values, $E_{r}$, for the inner and outer regions of the cortex can vary, with the $E_{r}$ values of the inner region slightly greater than those of the outer region.

In this work, Raman spectroscopy is used to investigate the protein secondary structures in the inner and outer regions of the feather cortex. Analysis of the Amide I region of Raman spectra taken from four birds (Swan, Gull, Mallard and Kestrel) shows that the $\beta$ sheet structural component decreases between the inner and outer region, relative to the protein side-chain components. This finding is consistent with the proposal that $E_{r}$ values are greater in the inner region than the outer region.

This work has shown that Raman spectroscopy can be used effectively to study the change in protein secondary structure between the inner and outer regions of a feather shaft. 


\section{Introduction}

Avian feathers are uniquely complex integumentary structures in vertebrates [1-3]. Feathers first evolved around 200 million years ago amongst theropod dinosaurs, and although not initially used for flight, these structures were first exapted for passive gliding and later for active flapping. Flapping flight means that modern bird feathers must not only be light but, also stiff enough and strong enough to withstand considerable aerodynamic loading. Biomechanical work has shown that the central shaft of a feather is 'overengineered'[4]; shaft safety factors are orders of magnitude higher than they need to be. Although all parts of a flight feather have been subject to evolutionary pressures to become stiff and strong, the central shaft is the part of the feather which resists the most load and exhibits some of the most remarkable mechanical properties. The central shaft is composed of two parts, the calamus and the rachis (Figure 1 ). The rachis, which comprises $\sim 75 \%$ of the shaft length, is the distal, extracutaneous part of the shaft to which vanes attach. It supports the vanes and exhibits complex cross-sectional geometry [5], with the diameter of the rachis tapering from the proximal end to the distal end. The shaft consists of a fibrous outer cortex and a foam-like core, the medulla. The fibrous cortex makes up the bulk of the shaft and has been shown to account for nearly all of its tensile strength when subject to longitudinal stresses [6,7]. If, on the other hand, the shaft is squeezed transversely, these stresses are resisted by the medulla $[8,9]$ and the cortex helps only by holding the core together. In application, then, the cortex resists bending loads and the medulla prevents buckling. The calamus is the remaining proximal region which inserts into the wing, it has a simple subcircular cross section and no medulla.

The major components of feathers are $\alpha$ - and $\beta$-keratin, which are encoded in multigene families [10]. $\beta$-keratin adds more rigidity than $\alpha$-keratin. Cellular and biochemical studies have shown that $\alpha$-keratin has played an important role in the early formation of the rachis, barbs, and barbules [10]. The exact structure of feather keratin remains unknown, although a reasonable model for the dominant protein secondary structure has been proposed based on X-ray diffraction data [11,12]. Results show that feather keratin is tightly packed in $\beta$-sheets into a coiled polypeptide chain that exhibits a high degree of disulphide cross-linkages, hydrophobic interactions, and hydrogen bonds [13,14]. This confers mechanical strength and chemical resistance, and the structure is largely insoluble due to the disulphide bridges. Structural studies have shown that in birds the $\beta$-keratin chain is typically 100-200 molecules long and contains a central conserved region of about 34 residues. This is believed to adopt a twisted antiparallel $\beta$-sheet conformation with three central strands and two partial outer strands $[11,15,16]$. Attached to the central region (or domain) are Amino-terminal and Carboxy-terminal domains. In the Amino-terminal domain, there are two subdomains (A and B) while in the Carboxy-terminal domain two subdomains ( $C$ and $D$ ) have been identified. Subdomain B is not present in birds. The central domain of the $\beta$-keratin chain is rich in $\beta$ and turn structures, and the rest of the structure contains mainly random coil units with some $\beta$-propensity. The most likely $\beta$-containing region in the feather shaft, apart from the conserved central 34-residue domain, is the variable length segment $\mathrm{C}$, with its potentially $\beta$-favouring sequence repeats $[15,17,18]$.

Up to $\sim 30 \%$ of the shaft length, the cortex has been shown to have two major layers; the thickest forms ca. 85\% of the cortex depth and directly overlies the medulla (Figure 1). The fibres in this layer are oriented along the shaft long axis and should support most of the load of the feather in application. There is also an outer layer which is the remaining cortical thickness (ca. 15\%), composed of fibres oriented at ca. $90^{\circ}$ to the long axis. Crossed-fibre 
architecture is well-established in animals $[19,20]$ as a way of providing extra strength and preventing damage. Further up the shaft, at greater than 30\% shaft length (in the rachis), there is only one layer and the fibres are axially oriented. This structural information has been derived from microbial biodegradation followed by scanning electron microscopy (SEM) measurements [3,21-23] , nanoindentation [24], CT scanning [24, 25], and polarised optical microscopy $[24,25]$. Observations with a microscope attached to the Raman spectrometer used in this work show this to be true of all feathers presented in this study.

Recently, we studied the shafts of a number of birds using nanoindentation [24]. The results, obtained for samples cut at $30 \%$ of the shaft length which had inner and outer layers, showed a clear mechanical anisotropy between the inner and outer layers which was caused by a change in fibre orientation. The reduced modulus, $E_{r}$, for the inner layer was greater than that for the outer layer when comparing nanoindentation measurements parallel to the fibre axis as well as when comparing them perpendicular to the fibre axis. Also, for the thicker inner layer, the reduced modulus $E_{r}$ for the inner part was greater than that for the outer part [24]. These changes might be explained by differences in protein secondary structures.

Infrared (IR) and Raman spectroscopy are two preferred methods currently used to investigate secondary protein structure [26-29] and are thus suitable tools to further investigate these $E_{r}$ observations. The vibrational signature of a protein amide group is very sensitive to polypeptide backbone conformation and, as a result, provides direct quantitative information regarding secondary structure. Differences in amide group geometric orientation and the environment for $\alpha$-helix, $\beta$-sheet, and $\beta$-turn as well as random coil structures in proteins lead to differences in amide vibrational absorptions. Raman spectroscopy also has several advantages over IR spectroscopy in the investigation of protein secondary structures. First, sampling is much easier and Raman is capable of much greater spatial resolution [30]. The Raman spectrometer used in this work was fitted with a confocal microscope which allowed areas of $\sim 4 \mu \mathrm{m} \times 27 \mu \mathrm{m}$ to be investigated, whereas this spatial sampling was not possible with the available IR spectrometer. Secondly, in addition to the characterisation and study of vibrational transitions of the amide groups, other bands can be seen more clearly in Raman spectra. This permits the environment of numerous amino acid side chains to be characterised and studied, notably acidic residues [31] and sulphur containing residues [32], including disulphide bonds (S-S units). Thirdly, Raman spectra are easily obtained from dilute samples in aqueous solution $[28,33]$ or samples which contain water. This is very difficult using IR spectroscopy as the water bending vibration absorption (at $\sim 1645 \mathrm{~cm}^{-1}$ ) obscures the main amide band (the Amide I band) in the IR spectrum. This is not a problem in Raman spectroscopy as the water bending mode has much lower intensity than in the IR spectrum.

Here, Raman spectroscopy is used to investigate the protein secondary structures within the inner and outer cortical regions of selected bird shafts. The aim is to address the following question: Do the different reduced modulus values of the inner and outer cortical regions correlate with differences in protein secondary structures within these regions, which contribute to their function and the mechanical properties in a feather shaft?

\section{Materials and Methods}

Bird material

Species were selected from across the avian phylogeny as different species require different material responses from their feathers. The feathers of a Gull in soaring flight, for example, do not behave in the same way as those of a flapping Swan or a hovering hummingbird. The birds used in this work encompassed a range of different flight styles and are as follows: Swan, Gull, Mallard, and Kestrel. The Swan, Cygnus olor and the Mallard, 
Anas platyrhynchos, are both Anseriformes. This is an ancient group of birds which diverged at the root of the avian family tree, the earliest part of this evolutionary radiation. However, they exist in very different flight niches. The Swan is among the heaviest of birds (Table 1) and requires very stiff feathers when it takes off from the surface of water because it is unable to jump. The Mallard only flies short distances and it does so by rapidly beating its wings. The common Gull (seagull), Larus canus, is a Charadriiform bird, which is another order nested at the root of the avian radiation. The Gull spends a lot of time at sea, in soaring flight and with wet feathers. The common Kestrel, Falco tinnunculus is a raptor (bird of prey) which evolved later and spends a lot of time hovering into oncoming wind before it dives for food.

Table 1

\section{Sample preparation}

The sample preparation method used here is very similar to that described in our earlier nanoindentation study [24]. The longest primary feathers (fourth-most distal) were excised from intact wings of four deceased birds. Those birds were the Mute Swan, Cygnus olor, the Common Kestrel, Falco tinnunculus, the Common Gull, Larus canus, and the Mallard, Anas platyrhynchos. $5 \mathrm{~mm}$ sections were removed with a Proxxon Minimot 40 hand-held rotary saw (Fohren, Germany) at $40 \%$ of the shaft length from the base. This was above the region where two layers were present in the cortex.

Replicate sections from the left and right wings were embedded in an 8:1 mix (resin: hardener, by weight) of Struers' EpoFix resin (Catcliffe, UK) inside a cylindrical silicone mould. Each sample was ground and polished through a series of lapidary papers to expose the shaft cross section, which is illustrated in Figure 1 . The surfaces were then given a final clean with a tissue soaked in solvent (ethanol) and stored in an enclosed Petri dish in air. The inner and outer layers of the cortex were observed in samples cut at up to $~ 30 \%$ of shaft length. For samples cut at longer lengths, only one layer, corresponding to the thicker inner layer observed at shorter lengths, was present.

In order to investigate Raman spectra recorded at different lengths along a shaft, the same procedure was repeated to prepare samples from nine feathers from three Swans. Sections were removed at 20\%, 40\%, 60\% and 80\% length of the first, third and fifth most distal feathers (P1, P3 and P5) of the right wing. 


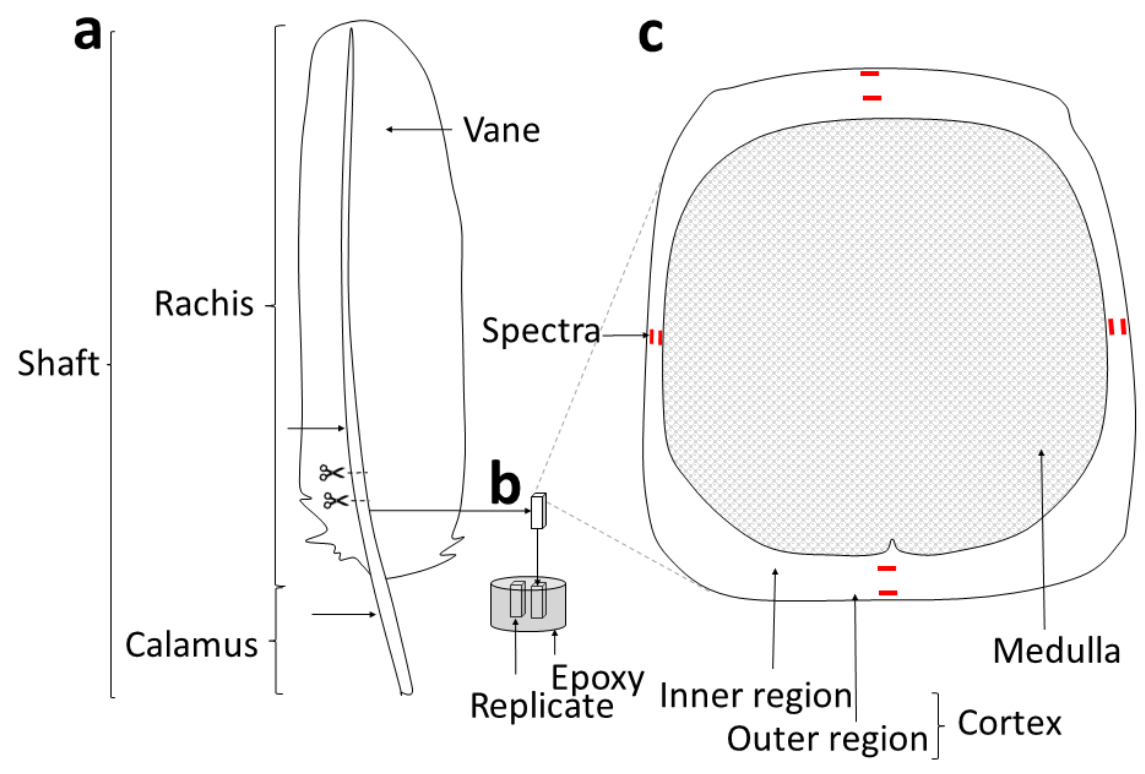

[Figure 1 (Schematic) - caption at bottom of document]

In some cases, a small gap formed between the feather shaft sample and the EPOfix resin. This is caused by bubbles in the resin because it shrinks slightly as it cures. In the worst cases, on exposure to the laser from the Raman spectrometer, this results in sample burning and saturation (even at low laser power) as well as fluorescence in less serious cases. The solution is either to grind down the surface past the region of the gap, or to infuse the surface with less viscous resin (such as MetPrep [Coventry, England] EPO-Set resin) under vacuum. In the worst cases a new sample was required (using MetPrep resin). Removal of bubbles from the matrix avoids the burning and fluorescence problem because the excited state responsible for the fluorescence readily loses energy to the matrix and the fluorescence is quenched. 


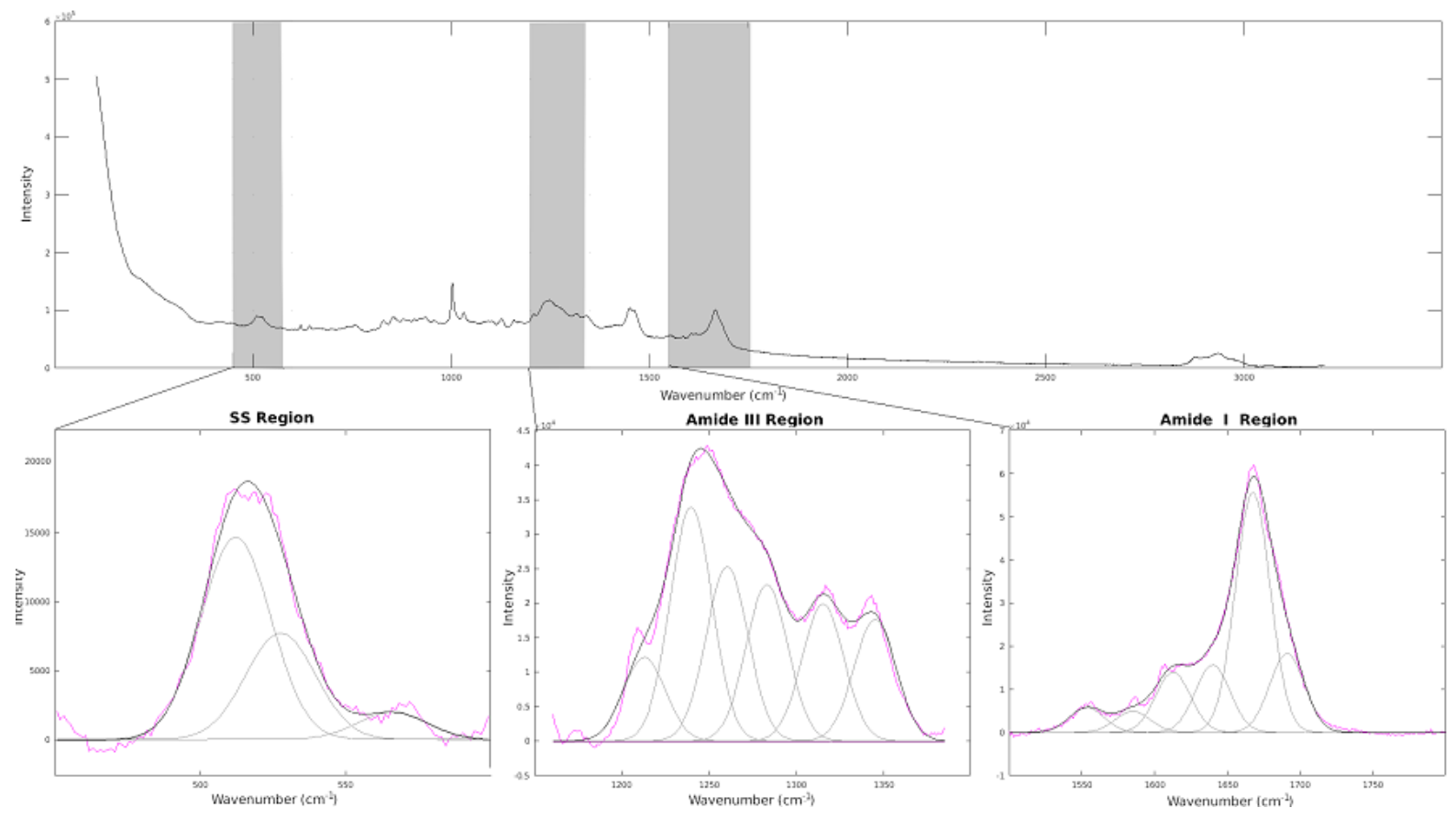

[ Figure 2 Example of a complete Raman spectrum]

\section{Raman spectroscopy}

Spectra were taken from the inner and outer regions, at dorsal, ventral, leading, and trailing positions, from both the left wing and the right wing, cut at $40 \%$ shaft length for each bird described in Table 1, as well as at $20 \%, 40 \%, 60 \%$, and $80 \%$ of the shaft length for the right wings of the nine Swan feathers in the dorsal and ventral positions. This was done using a Renishaw InVia Confocal Raman Microscope (Wotton-under-Edge, UK) equipped with a $100 \mathrm{~mW}$, diode laser at $785 \mathrm{~nm}$ wavelength and with a $\times 50 / 0.75$ objective (50 is the magnification and 0.75 is the numerical aperture). The line-focused laser is $(4 \pm 1) \mu \mathrm{m}$ wide and $(27 \pm 2) \mu \mathrm{m}$ long on the sample. Two layers could be clearly seen, for samples cut at $20 \%$ length, and the laser was positioned in the middle of each layer, using the XY stage and the microscope eyepiece. For samples where only one layer was observed (samples cut at > $\sim 30 \%$ shaft length) the laser was positioned close to the inner (or outer) edge of the cortex. The spectrometer was calibrated using a silicon standard before each session. Spectra were recorded between 3,200 and $100 \mathrm{~cm}^{-1}$. A total of eight accumulations were taken for each sample at $10 \mathrm{~mW}$ with 60 seconds acquisition time for each accumulation. These parameters (power, number of accumulations, acquisition time) were selected after recording test spectra in the Amide I and III spectral regions using a range of conditions. The selection of final parameters to be used was based on the resolution and signal-to-noise in these test spectra, and comparison of $R^{2}$ values once the spectra had been fitted ( $R$ is the correlation coefficient). More information on the Raman spectrometer and the selection of parameters used to acquire spectra is given in the Supplementary Information (SI) section.

Table 2 [Amide 1 band assignments] 


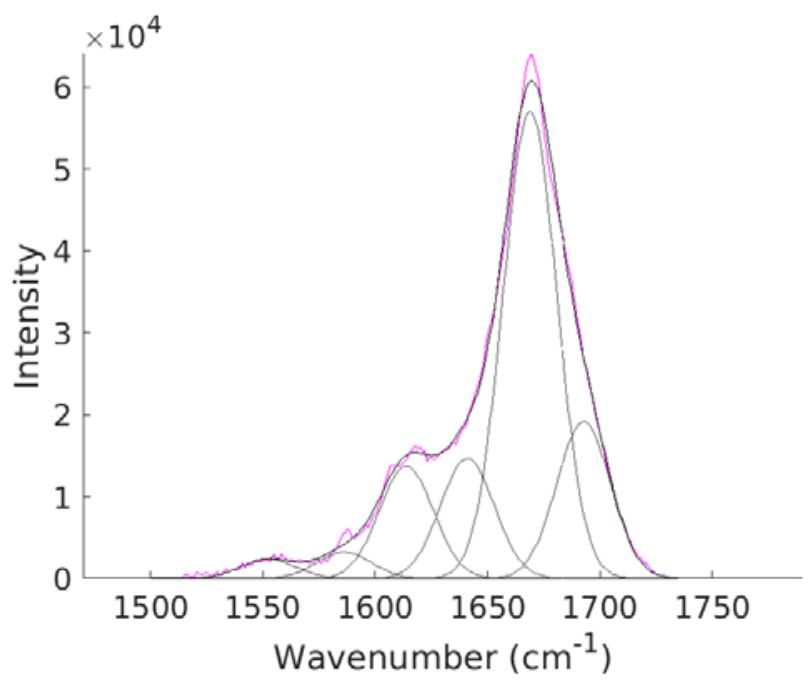

[Figure 3 (Amide 1 fit) - caption at bottom of document]

\section{Spectral Regions Investigated and Band Fitting}

In the experimental spectra obtained between 3,200 and $100 \mathrm{~cm}^{-1}$ (see Figure 2), particular attention was given to the Amide I region (1560-1800 $\mathrm{cm}^{-1}$ ), the Amide III region (1200-1350 cm $\mathrm{cm}^{-1}$ ), and the disulphide bond (S-S) region (400-600 $\left.\mathrm{cm}^{-1}\right)$. Assignments made in these regions follow those in previous work [27,29,34-41]. See Tables 2-4.

The Amide I region arises from C-O stretching vibrations. This provides information on protein secondary structure in the samples investigated [29,34,36-39]. The Amide III region (1200-1350 $\mathrm{cm}^{-1}$ ) arises from a combination of $\mathrm{N}-\mathrm{H}$ bending and $\mathrm{C}-\mathrm{N}$ stretching vibrational modes. Previously, this region has also been used to investigate protein secondary structure $[13,27,38,40]$, notably in cases where infrared spectra were recorded from proteincontaining samples in solution, where the water bending mode absorption overlaps with the Amide I region and makes the Amide I region of limited use. However, in the present work, Raman spectra were recorded for solid samples embedded in a resin matrix and in any case, the water bending absorption is much less intense in Raman spectroscopy than in infrared spectroscopy. Hence both the Amide I and Amide III regions were investigated in this work. The Amide II region (1510-1560 $\left.\mathrm{cm}^{-1}\right)$ is also related to transitions of combinations of $\mathrm{N}-\mathrm{H}$ bending and $\mathrm{C}-\mathrm{N}$ stretching modes. However, the transitions observed in this region correspond mainly to change in the H-bonding environment and the Amide II bands are often overlapped by bands originating from amino acid side chain vibrations [27,34,42-44]. Hence, this region is usually not used for investigating protein secondary structure because the correlation between secondary structure and band position is much harder to establish than in the Amide I and Amide III regions. The disulphide bond (S-S) region provides information on the CC-S-S-CC conformation of disulphide bonds in keratin [36], specifically, the conformations around the S-S bonds in the cystine groups in the protein. These bands are centred at 510, 525 and $560 \mathrm{~cm}^{-1}$. The $510 \mathrm{~cm}^{-1}$ and $525 \mathrm{~cm}^{-1}$ vibrations arise from the CCS-S-CC band conformations Gauche-Gauche-Gauche (G-G-G) and Gauche-Gauche-Trans (G-G-T) respectively and the $560 \mathrm{~cm}^{-1}$ transition arises from a sulphur-sulphur stretching mode coupled with a cystine residue absorption [35,36,41]. It was notable that a vibration was expected at $540 \mathrm{~cm}^{-1}$ (assigned to Trans-Gauche-Trans (T-G-T)), but this was not observed in any of the samples investigated. 
Table 3 [Amide 3 band assignments]

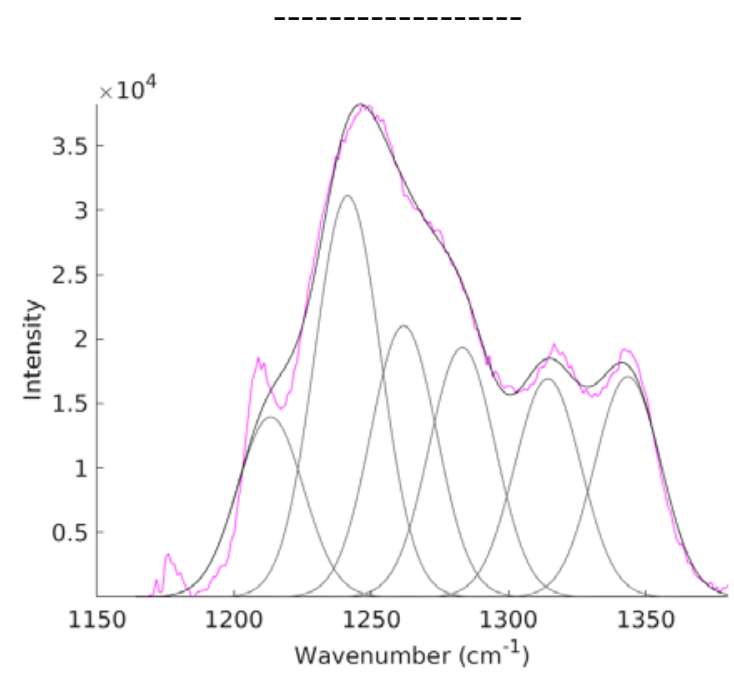

[Figure 4 (Amide III fit) - caption at bottom of document]

Experimental spectra were baseline corrected and fitted using a script generated using the MATLAB curve fitting toolbox. Following work by other researchers [27,29,34-41], Gaussian bands were used in the fitting (with a half-width of $28.2 \mathrm{~cm}^{-1}$ ). The number of Gaussian bands used to fit each region investigated was chosen based on the results of trial fits with different numbers of bands, as well as fits performed in these regions by other research groups [27,29,34-41]. The Amide I region was fitted with six Gaussian bands. A typical fit is shown in Figure 3 and a summary of the band assignments made in this region, along with those from previous work is shown in Table 2 [29,34,36-39]. The same fitting procedure, also using six bands with the same half-width, was used for the Amide III region. A typical fit in this region is shown in Figure 4 and band assignments are shown in Table 3 $[13,27,38,40]$. Figure 5 shows a typical fit from the S-S region, which was fitted with 3 Gaussian bands, again with a half-width of $28.2 \mathrm{~cm}^{-1}$. Band assignments used in this work are listed in Table 4 and follow those of refs $[35,36,41]$. Each band intensity ratio was quoted with an error which is derived from the 95\% confidence interval of each fit. An explanation for the choice of band half-width as $28.2 \mathrm{~cm}^{-1}$ is given in the SI section.

Table 4 [SS band assignments] 


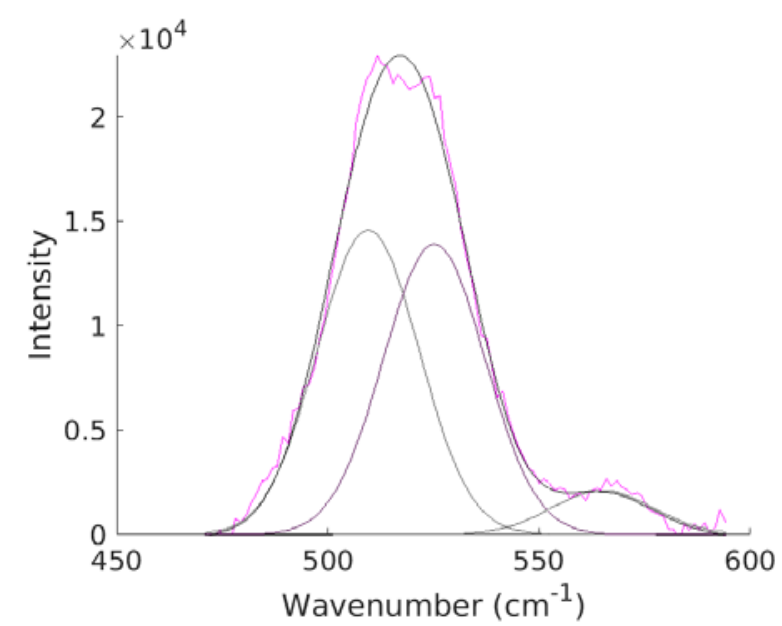

[Figure 5 (S-S fit) - caption at bottom of document]

\section{Results and Discussion}

In order to investigate possible differences in protein secondary structure between the inner and outer regions of the cortices of the shaft samples, band intensity ratios were measured in the Amide I, Amide III and disulphide bond (S-S) regions for the four selected birds. For the Amide I region (1560-1800 $\mathrm{cm}^{-1}$ ), the six-band fitting method used followed the fits carried out by other groups in this spectral region [27,29,34$37,40,41]$. This method gave a good fit to the experimental spectra (Figure 3 ). The recommended assignments, which are presented in Table 2 (column_-68), were made based on a review of the assignments made in relevant recent literature [29,34,36,39]. The six fitted bands in the Amide III region (1200-1350 $\mathrm{cm}^{-1}$ ) (Figure 4) and the three fitted bands in the S-S (400-600 $\left.\mathrm{cm}^{-1}\right)$ (Figure 5) were assigned in the same way, see Table 3 (column 56) and Table 4 (column 5) respectively. 

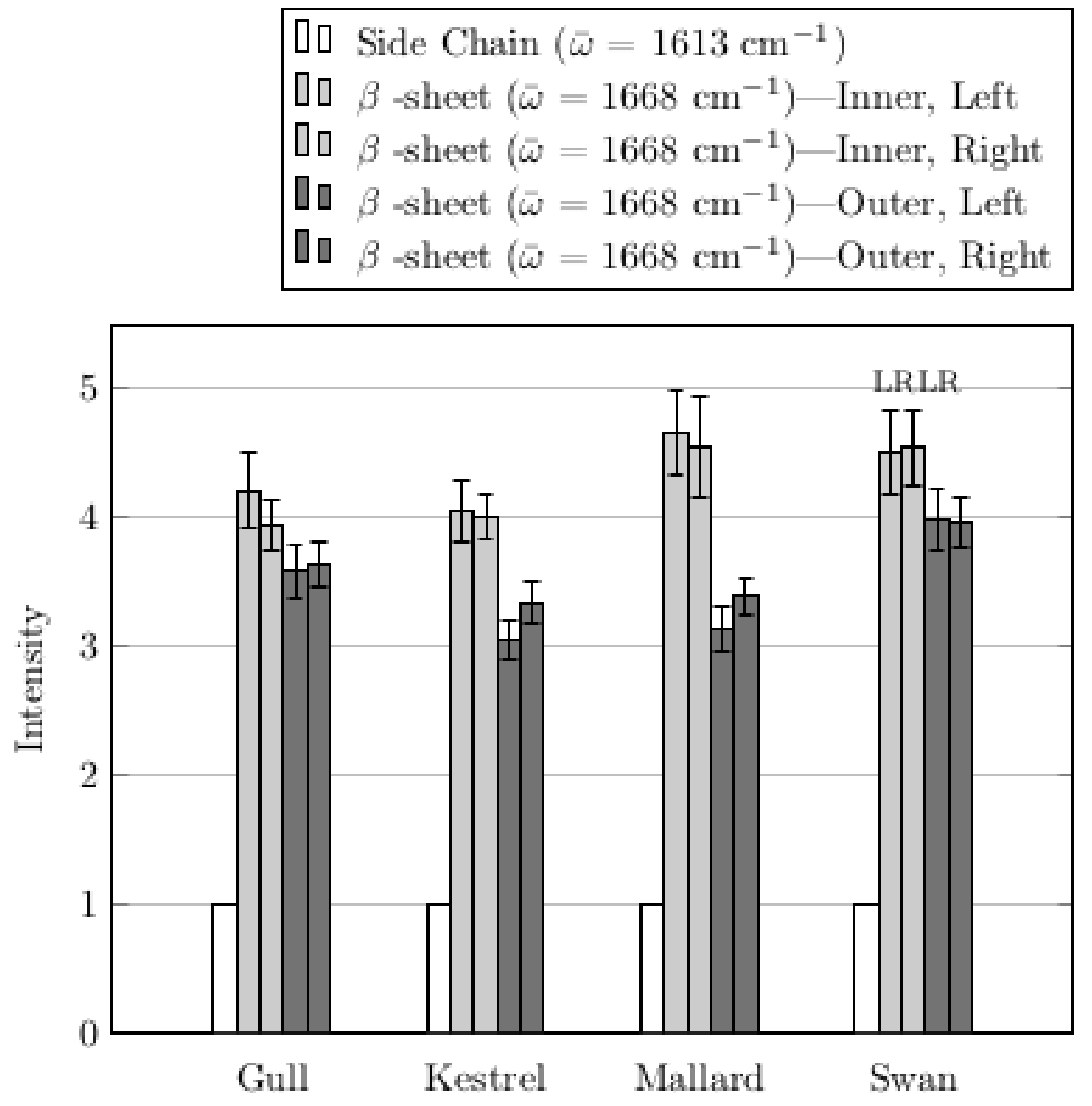

[Figure 6 (Amide I band intensity ratios (a)) - caption at bottom of the document]

Figure 6 shows an example of band intensity ratios obtained in the Amide I region for a Gull, Kestrel, Mallard and Swan for samples cut at $40 \%$ length. It shows the intensity ratios for the bands at $1668 \mathrm{~cm}^{-1}$ and $1613 \mathrm{~cm}^{-1}$, which are $\beta$-sheet and side-chain bands respectively. For this figure, values for feathers from the left $(\mathrm{L})$ and right $(\mathrm{R})$ wings of the same bird are shown. This is indicated in the figure by the characters ' $L$ ' and ' $R$ '. The ratio decreases for all four birds on going from the inner to the outer region. Figure 7 shows an example of band intensity ratios obtained in the Amide III region for these Gull, Kestrel, Mallard, and Swan samples. It shows the band intensity ratios for the bands at $1262 \mathrm{~cm}^{-1}$ and $1240 \mathrm{~cm}^{-1}$, which are random coil and $\beta$-sheet bands respectively. It can be seen that the experimental errors are greater in Figure 7 than Figure 6, and as a result, no clear trend can be seen in Figure 7, although based on the trend seen in Figure 6 an increase in the band intensity ratio $1262 \mathrm{~cm}^{-1}: 1240 \mathrm{~cm}^{-1}$ (random coil: $\beta$-sheet) might have been expected on going from the inner to the outer region.

A Raman spectrum in the S-S region recorded for the shaft of the left wing of a Mallard cut at $40 \%$ length is presented in Figure 5, above. Three bands were fitted to this spectrum with band maxima of 525, 540 and $560 \mathrm{~cm}^{-1}$, which correspond to two different conformations of a CC-S-S-CC unit in cystine groups [36](G-G-G and G-G-T) and an S-S stretch coupled with a cystine residue absorption respectively [36]. The ratio of the G-G-G conformation to the total disulphide bonds has been suggested as a criterion for the stability 
of disulphide groups in keratin $[35,45]$ and this would indicate that the band intensity ratio $510 \mathrm{~cm}^{-1}: 525 \mathrm{~cm}^{-1}$ should decrease on going from the inner to the outer region. Unfortunately, no trend could be observed for this ratio on going from the inner to the outer region as the experimental errors are large.

As the signal-to-noise was better in the Amide I region than the Amide III and S-S regions, the experimental band intensity ratios in the Amide I region showed the lowest experimental errors compared to those in the other two regions (compare for example the experimental errors in Figures 6 and 7). As a result, most attention was given to band intensity ratios in the Amide I region to investigate possible differences in protein structure between the inner and outer regions. Inspection of the Amide I spectrum in Figure 3 shows that the bands at 1553 and $1585 \mathrm{~cm}^{-1}$ (assigned to ring modes) are much weaker than the other bands at 1613, 1640, 1668 and $1692 \mathrm{~cm}^{-1}$. This was also found in all Amide I spectra recorded. Therefore, only the four more intense bands were used to determine band intensity ratios, with the intensity of three bands being evaluated relative to the intensity of the 1613 $\mathrm{cm}^{-1}$ band i.e. the following ratios were evaluated: (a) $1668 \mathrm{~cm}^{-1}: 1613 \mathrm{~cm}^{-1} \beta$-sheet: side chain, (b) $1640 \mathrm{~cm}^{-1}: 1613 \mathrm{~cm}^{-1} \alpha$-helix: side chain and (c) $1692 \mathrm{~cm}^{-1}: 1613 \mathrm{~cm}^{-1} \beta$-turn: sidechain. Of these ratios, (a) which involves the $\beta$-sheet band at $1668 \mathrm{~cm}^{-1}$ might be expected to show the largest change if there is a change in protein structure between the inner and outer regions (e.g. an increase in $\beta$-sheets from $\beta$-keratin) as $\beta$-keratin is known to provide more rigidity than $\alpha$-keratin [10]. This proved to be the case on going from the inner to the outer regions. Examples of the change of the ratio (a) are shown in Figures 6. These observed trends were the same for all four birds with all three ratios decreasing from the inner to the outer region. These results indicate that there is more $\beta$-sheet in the inner region, than the outer region of the cortex, relative to side-chain groups. There was no significant change in ratios (b) and (c) on going from the inner to the outer regions, taking into account experimental errors (see the SI section).

Although we have observed that the $\beta$-sheet component is larger in the inner region than the outer region, it is difficult to determine the reason for this in terms of change in keratin structure. An increase of $\beta$-keratin in the inner region, relative to the outer region, may reflect an increase in segment $C$ in the inner region (caused by larger $C$ segments in the different $\beta$-keratins present) and/or a greater $\beta$-content in the $C$ segments in the same proteins $[15,17,18]$. It might also be caused by different relative fractions of fibre: matrix in each region.

Band intensity ratios were also measured in the Amide I, Amide III and S-S regions for the outer regions of the shafts of the four birds studied in this work at the dorsal, ventral, trailing and leading positions at $40 \%$ of the rachis length from the base. Again particular attention was given to ratios measured in the Amide I region. For the bands (a)-(c) measured for these four positions, no trend in the ratios (a) - (c) could be observed between these positions and, within experimental error, each ratio was the same in the four positions.

The Amide I band intensity ratios (a) - (c) were also investigated at different positions along the length of selected shaft samples from Swan feathers, at the dorsal and ventral positions. Spectra were taken from cross-sections of Swan shafts at 20, 40,60, and 80\% of the length from the base of the shaft at the dorsal and ventral positions. Nine feathers were investigated - three feathers (P1, P3 and P5) from the right wings of three Swans (Cygnus spp.). Figure 8 shows an example of results obtained for band intensity ratio. Inspection of all the results indicates that there is a decrease in ratio (a) on going from the inner to the outer regions, but there are no significant changes in these ratios along the length of a shaft or between the dorsal and ventral positions. 
The observed change in ratio (a) from the inner to the outer region of a shaft apply to all four of the birds studied and could therefore represent the first report of a new neornithine synapomorphy as the trait is observed in a basal order Anseriformes (Swan, Mallard) as well the derived order Falconiformes (Kestrel), though more work would need to be done on other groups to confirm a neornithine synapomorphy. This would imply that bird feathers adapted to a selection pressure before the groups investigated in this paper, or perhaps all extant birds, diverged from the root of the avian phylogeny.

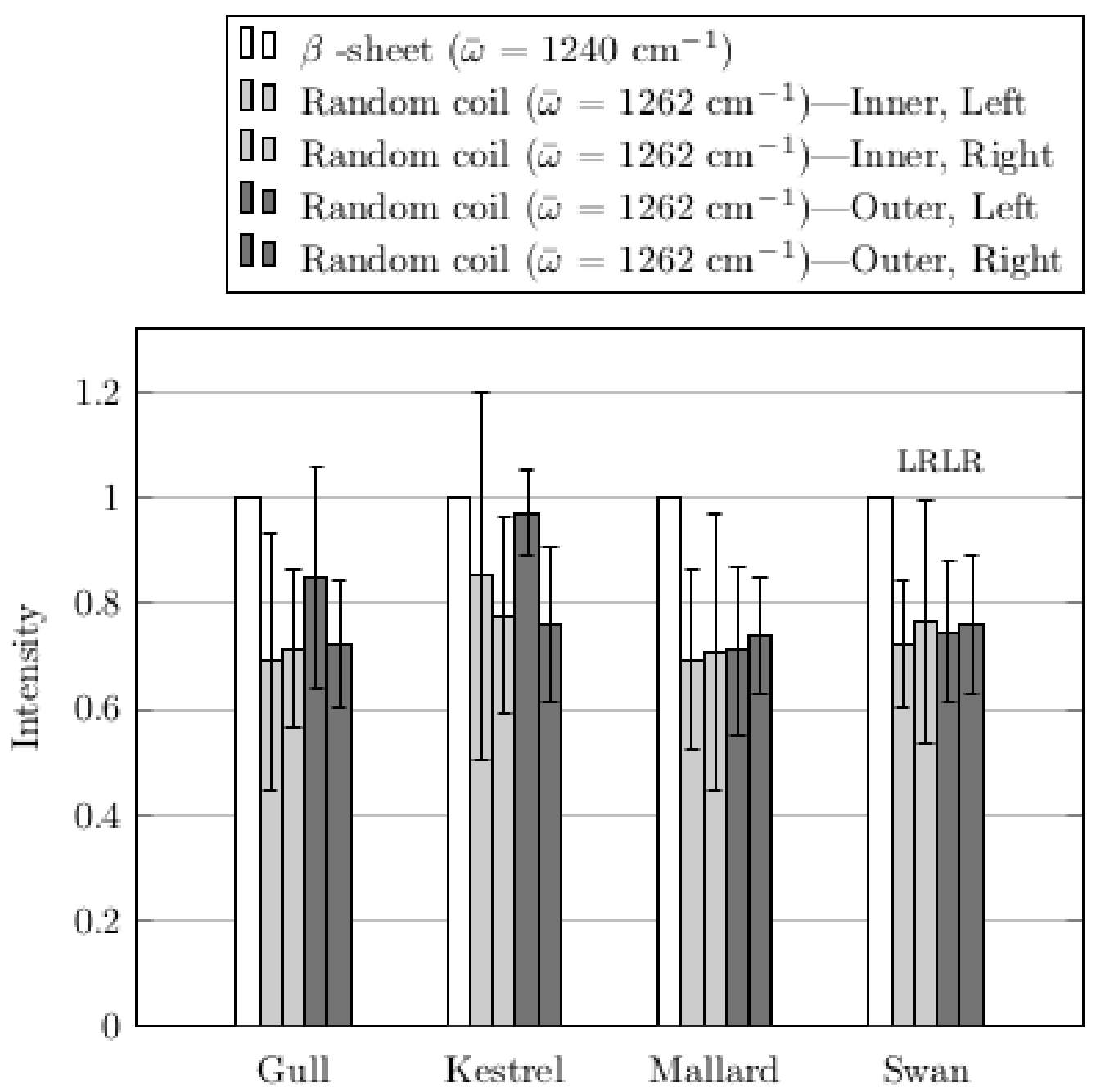

[Figure 7---(example of Amide III band intensity ratios) -caption at bottom of the document] 


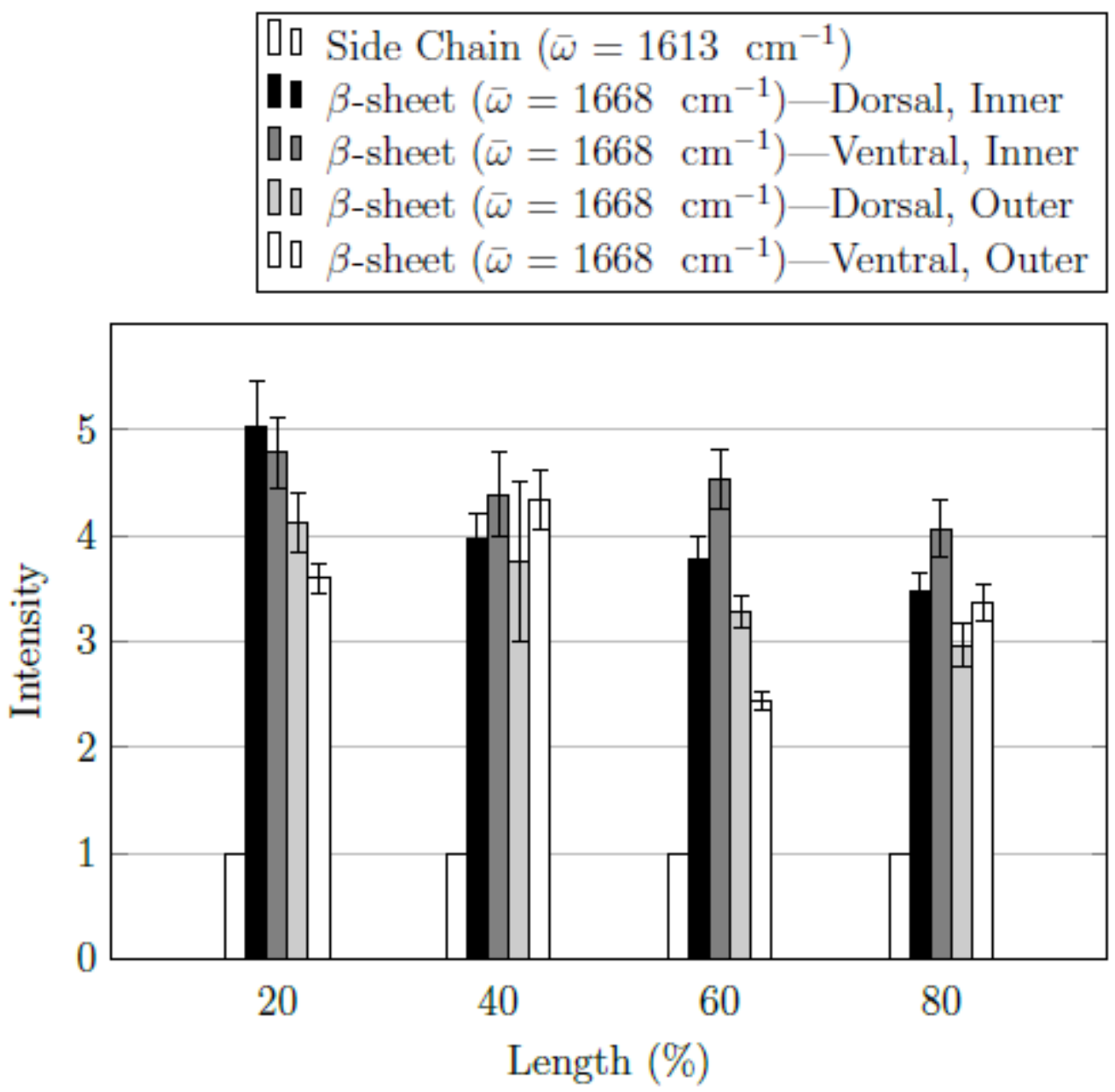

[Figure 8--(Amide I band intensity ratios (a) along the length of a Swan rachis sample, caption at bottom of the document] 


\section{Conclusions}

In this work, Raman spectra were recorded from the inner and outer regions of feather shaft cross-sections of four birds; Swan, Gull, Mallard and Kestrel. Spectra were obtained in the range 3200-100 $\mathrm{cm}^{-1}$ and were analysed in three regions; Amide I (1560-1800 $\mathrm{cm}^{-1}$ ), Amide III (1200-1350 $\left.\mathrm{cm}^{-1}\right)$ and S-S $\left(400-600 \mathrm{~cm}^{-1}\right)$. For each sample investigated, the Amide I region showed the best signal-noise and information on the protein secondary structures in the inner and outer regions of the cortex were obtained from these measurements. It was concluded that the $\beta$-sheet component is larger in the inner region than the outer region relative to the protein side chain components. This is consistent with the proposal that $E_{r}$ values are greater in the inner region than the outer region. This trend was also observed along the length of a shaft, but no significant difference in this behaviour was observed at different distances from the base of the shaft or between dorsal or ventral positions.

Therefore, in answering the question - do the different reduced modulus values of the inner and outer cortical regions correlate with differences in the protein secondary structures within these regions? We conclude that differences in protein secondary structure between regions can be observed and they would contribute to the difference in properties of these regions. It also makes sense in application for the load bearing inner region to be stiffer than the outer region which would prevent buckling. However, this investigation does not observe any differences in sections cut at different lengths along a shaft or between different feathers on the wing.

In summary, this work has shown that Raman spectroscopy can be used effectively to study the change in protein secondary structure of the inner and outer regions of a feather shaft. It is anticipated that with improvement of the technique and sample preparation methods that more detail will be derived concerning the protein secondary structures in these regions. It would certainly be advantageous if the Amide III region, as well as the S-S region, could be used with the Amide I region, with an internal standard band such as the side-chain band in the Amide I region to monitor changes of protein secondary structure. 


\section{Table 1:}

The birds used in this study.

\begin{tabular}{l|l|l|l|l} 
Common Name & Binomial name & Flight style & Order & $\begin{array}{l}\text { Typical } \\
\text { weight } \\
\text { (g) }\end{array}$ \\
\hline & & & & \\
\hline Gull & Larus canus & Soaring & Charadriiformes & 430 \\
\hline Kestrel & Falco tinnunculus & $\begin{array}{l}\text { Hovering } \\
\text { Soaring }\end{array}$ & Falconiformes & 180 \\
\hline Mallard & $\begin{array}{l}\text { Anas } \\
\text { platyrhynchos }\end{array}$ & $\begin{array}{l}\text { Continuous } \\
\text { flapping }\end{array}$ & Anseriformes & 1000 \\
\hline Swan & Cygnus lour & Fast flapping & Anseriformes & 10,000
\end{tabular}

Table 2

Summary of Amide I band assignments in recent literature and in this work (see text).

\begin{tabular}{l|l|l|l|l|l}
$\begin{array}{c}\overline{\mathbf{\omega}} \\
\left(\mathbf{c m}^{-\mathbf{1}}\right)\end{array}$ & ref. [36] & ref. [37] & ref. [29] & ref. [39] & Recommendation \\
\hline 1553 & & & & & Ring mode \\
\hline 1585 & & & & & Ring mode \\
\hline 1613 & & & Side chain & $\begin{array}{l}\text { Side } \\
\text { chain }\end{array}$ & $\begin{array}{l}\text { Side chain, } \\
\text { Ring mode, }\end{array}$ \\
\hline 1640 & & $\alpha$-helix & & $\alpha$-helix & $\alpha$-helix \\
\hline 1668 & $\beta$-sheet & $\beta$-sheet & $\beta$-sheet & $\beta$-sheet & $\beta$-sheet \\
\hline 1692 & & & $\beta$-turn & & $\beta$-turn
\end{tabular}


Table 3

Summary of the Amide III band assignments in recent literature and in this work (see text).

\begin{tabular}{l|l|l|l|l}
$\begin{array}{c}\overline{\boldsymbol{\omega}} \\
\left(\mathbf{c m}^{-1}\right)\end{array}$ & ref. [27] & ref. [40] & ref. [13] & Recommendation \\
\hline 1214 & $\beta$-sheet & & & Ring Mode \\
\hline 1240 & $\beta$-sheet & $\beta$-sheet & $\beta$-sheet & $\beta$-sheet \\
\hline 1262 & Random coil & Random coil & $\begin{array}{l}\text { Random } \\
\text { coil }\end{array}$ & Random coil \\
\hline 1283 & $\beta$-turn & $\beta$-turn & $\beta$-turn & $\beta$-turn \\
\hline 1313 & $\alpha$-helix & $\alpha$-helix & $\alpha$-helix & $\alpha$-helix \\
\hline 1341 & & $\alpha$-helix & & $\alpha$-helix
\end{tabular}

\section{Table 4}

Summary of the S-S region band assignments in the recent literature (Abbreviations: G, Gauche; T, Trans) and in this work (see text).

Labels G-G-G and T-G-T are used to describe CC-S-S-CC conformations around a disulphide bond in a keratin protein in a rachis sample; see [36].

\begin{tabular}{l|l|l|l|l}
$\begin{array}{c}\overline{\mathbf{\omega}} \\
\left(\mathbf{c m}^{-\mathbf{1}}\right)\end{array}$ & ref. [36] & ref. [41] & ref. [35] & Recommendation \\
\hline 493 & & & G-G-G & \\
\hline 510 & G-G-G & G-G-G & G-G-T & G-G-G \\
\hline 525 & G-G-T & G-G-T & & T-G-T G-G-T \\
\hline 540 & T-G-T & & $\begin{array}{l}\text { S-S stretch and } \\
\text { cystine residue } \\
\text { absorption }\end{array}$ & $\begin{array}{l}\text { S-S stretch and } \\
\text { cystine residue } \\
\text { absorption }\end{array}$
\end{tabular}




\section{Acknowledgements}

CL was supported by the Natural Environmental Research Council [grant number NE/L002531/1] and the Engineering and Physical Sciences Research Council (EPSRC). CL and GD were supported by UEFISCDI Romania [grant number PN-III-P4-ID-PCE-20160572].

The authors thank Dr John Chitty of Anton Vets and Slimbridge Wildlife park for providing bird carcasses, and Professor Andrea Russell and her group (Southampton University) for advice on using the Raman spectrometer.

\section{References}

1. Lucas AM, Stettenheim PR. 1972 Avian anatomy: Integument (Agricultural Handbook 362). Michigan: United States Department of Agriculture.

2. Chuong C-M, Chodankar R, Widelitz RB, Jiang T-X. 2000 Evo-Devo of feathers and scales: building complex epithelial appendages. Curr. Opin. Genet. Dev. 10, 449-56. (doi:10.1016/S0959-437X(00)00111-8)

3. Lingham-Soliar T. 2013 Feather structure, biomechanics and biomimetics: the incredible lightness of being. J. Ornithol. 155, 323-336. (doi:10.1007/s10336-0131038-0)

4. Wang X, Nudds RL, Palmer C, Dyke G. 2017 Primary feather vane asymmetry should not be used to predict the flight capabilities of feathered fossils. Sci. Bull. 62, 12271228. (doi:10.1016/j.scib.2017.08.025)

5. Bachmann T, Emmerlich J, Baumgartner W, Schneider J, Wagner H. 2012 Flexural stiffness of feather shafts: geometry rules over material properties. J. Exp. Biol. 215, 405-15. (doi:10.1242/jeb.059451)

6. Weiss IM, Kirchner HOK. 2011 Plasticity of two structural proteins: alpha-collagen and beta-keratin. J. Mech. Behav. Biomed. Mater. 4, 733-43.

(doi:10.1016/j.jmbbm.2011.02.008)

7. Purslow P, Vincent JF V. 1978 Mechanical properties of primary feathers from the pigeon. J. Exp. Biol. 72, 251-260.

8. Zou M, Zhou J, Xu L, Song J, Liu S, Li X. 2019 An engineering perspective on the microstructure and compression properties of the seagull Larus argentatus feather rachis. Micron 126, 102735. (doi:10.1016/J.MICRON.2019.102735)

9. Wang B. 2016 Structural and functional design strategies of biological keratinous materials. UC San Diego. See http://escholarship.org/uc/item/9pj4f2wv.

10. Alibardi L, Toni M. 2006 Cytochemical, biochemical and molecular aspects of the process of keratinization in the epidermis of reptilian scales. Prog. Histochem. Cytochem. 40, 73-134. (doi:10.1016/j.proghi.2006.01.001)

11. Fraser RDB, Parry DAD. 2011 The structural basis of the filament-matrix texture in 
the avian/reptilian group of hard a-keratins. J. Struct. Biol. 173, 391-405. (doi:10.1016/j.jsb.2010.09.020)

12. Fraser RDB, Parry DAD. 2008 Molecular packing in the feather keratin filament. $J$. Struct. Biol. 162, 1-13. (doi:10.1016/j.jsb.2008.01.011)

13. Zhao W, Yang R, Zhang Y, Wu L. 2012 Sustainable and practical utilization of feather keratin by an innovative physicochemical pretreatment: high density steam flashexplosion. Green Chem. 14, 3352. (doi:10.1039/c2gc36243k)

14. Pabisch S, Puchegger S, Kirchner HOK, Weiss IM, Peterlik H. 2010 Keratin homogeneity in the tail feathers of Pavo cristatus and Pavo cristatus mut. alba. $J$. Struct. Biol. 172, 270-275. (doi:10.1016/j.jsb.2010.07.003)

15. Bruce-Fraser RD B , Parry DAD. 2020 Lepidosaur ß-Keratin Chains With Four 34Residue Repeats: Modelling Reveals A Potential Filament-Crosslinking Role. J. Struct. Biol. , 107413. (doi:10.1016/J.JSB.2019.107413)

16. Calvaresi M, Eckhart L, Alibardi L. 2016 The molecular organization of the beta-sheet region in Corneous beta-proteins (beta-keratins) of sauropsids explains its stability and polymerization into filaments. J. Struct. Biol. 194, 282-291.

(doi:10.1016/j.jsb.2016.03.004)

17. Parry DAD, Fraser RDB, Alibardi L, Rutherford KM, Gemmell N. 2019 Molecular structure of sauropsid $\beta$-keratins from tuatara (Sphenodon punctatus). J. Struct. Biol. 207, 21-28. (doi:10.1016/j.jsb.2019.04.008)

18. Fraser RDB, Parry DAD. 2014 Amino acid sequence homologies in the hard keratins of birds and reptiles, and their implications for molecular structure and physical properties. J. Struct. Biol. 188, 213-224. (doi:10.1016/j.jsb.2014.10.012)

19. Shadwick RE. 2008 Foundations of animal hydraulics: geodesic fibres control the shape of soft bodied animals. J. Exp. Biol. 211, 289-91. (doi:10.1242/jeb.008912)

20. Alexander RM. 1987 Bending of cylindrical animals with helical fibres in their skin or cuticle. J. Theor. Biol. 124, 97-110. (doi:10.1016/S0022-5193(87)80255-2)

21. Lingham-Soliar T. 2017 Microstructural tissue-engineering in the rachis and barbs of bird feathers. Sci. Rep. 7, 45162. (doi:10.1038/srep45162)

22. Lingham-Soliar T, Bonser RHC, Wesley-Smith J. 2010 Selective biodegradation of keratin matrix in feather rachis reveals classic bioengineering. Proc. Biol. Sci. 277, 1161-8. (doi:10.1098/rspb.2009.1980)

23. Lingham-Soliar T, Murugan N. 2013 A new helical crossed-fibre structure of $\beta$-keratin in flight feathers and its biomechanical implications. PLoS One 8, e65849. (doi:10.1371/journal.pone.0065849)

24. Laurent CM, Palmer C, Boardman RP, Dyke G, Cook RB. 2014 Nanomechanical properties of bird feather rachises: Exploring naturally occurring fibre reinforced laminar composites. J. R. Soc. Interface 11. (doi:10.1098/rsif.2014.0961)

25. (a) Laurent CM, Ahmed SI, Boardman RP, Cook, R.B., Dyke G, Palmer C, Schneider P, DeKat R. 2019 Imaging techniques for observing laminar geometry in the feather shaft cortex. J. Microsc. , jmi.12820. (doi:10.1111/jmi.12820)

(b) Laurent CM unpublished work

26. Barth A. 2007 Infrared spectroscopy of proteins. Biochim. Biophys. Acta - Bioenerg. 
1767, 1073-1101. (doi:10.1016/J.BBABIO.2007.06.004)

27. Cai S, Singh BR. 1999 Identification of $\beta$-turn and random coil amide III infrared bands for secondary structure estimation of proteins. Biophys. Chem. 80, 7-20. (doi:10.1016/S0301-4622(99)00060-5)

28. Pelton JT, McLean LR. 2000 Spectroscopic Methods for Analysis of Protein Secondary Structure. Anal. Biochem. 277, 167-176. (doi:10.1006/ABIO.1999.4320)

29. Hahn MB et al. 2015 Influence of the Compatible Solute Ectoine on the Local Water Structure: Implications for the Binding of the Protein G5P to DNA. J. Phys. Chem. B 119, 15212-15220. (doi:10.1021/acs.jpcb.5b09506)

30. Rintoul L, Carter EA, Stewart SD, Fredericks PM. 2000 Keratin orientation in wool and feathers by polarized Raman spectroscopy. Biopolym. - Biospectroscopy Sect. 57, 19-28. (doi:10.1002/(SICI)1097-0282(2000)57:1<19::AID-BIP4>3.0.CO;2-Z)

31. Overman SA, Jr. GJT. 1999 Raman Markers of Nonaromatic Side Chains in an $\alpha-$ Helix Assembly: Ala, Asp, Glu, Gly, Ile, Leu, Lys, Ser, and Val Residues of Phage fd Subunits†,‡. Biochemistry 38, 4018-4027. (doi:10.1021/BI982901E)

32. Rajkumar BJ., Ramakrishnan V. 2001 Vibrational spectroscopic study of dlmethionine dihydrogen phosphate. Spectrochim. Acta Part A Mol. Biomol. Spectrosc. 57, 247-254. (doi:10.1016/S1386-1425(00)00355-3)

33. Dong J, Dinakarpandian D, Carey PR. 1998 Extending the raman analysis of biological samples to the 100 micromolar concentration range. Appl. Spectrosc.

34. Zhang Q, Liebeck BM, Yan K, Demco DE, Körner A, Popescu C. 2012 Alpha-Helix Self-Assembly of Oligopeptides Originated From Beta-Sheet Keratin. Macromol. Chem. Phys. 213, 2628-2638. (doi:10.1002/macp.201200446)

35. Essendoubi M, Meunier M, Scandolera A, Gobinet C, Manfait M, Lambert C, Auriol D, Reynaud R, Piot O. 2019 Conformation changes in human hair keratin observed using confocal Raman spectroscopy after active ingredient application. Int. J. Cosmet. Sci. , 12528. (doi:10.1111/ics.12528)

36. Akhtar W, Edwards HG. 1997 Fourier-transform Raman spectroscopy of mammalian and avian keratotic biopolymers. Spectrochim. Acta. A. Mol. Biomol. Spectrosc. 53, 81-90. (doi:10.1016/S1386-1425(97)83011-9)

37. Blanch EW, Hecht L, Barron LD. 2003 Vibrational Raman optical activity of proteins, nucleic acids, and viruses. Methods 29, 196-209. (doi:10.1016/S1046-2023(02)003109)

38. Fu F-N et al. 1994 Secondary Structure Estimation of Proteins Using the Amide III Region of Fourier Transform Infrared Spectroscopy: Application to Analyze CalciumBinding-Induced Structural Changes in Calsequestrin. Appl. Spectrosc. Vol. 48, Issue 11, pp. 1432-1441 48, 1432-1441. (doi:10.1366/0003702944028065)

39. Maiti N, Apetri MM, Zagorski MG, Carey PR, Anderson VE. 2004 Raman Spectroscopic Characterization of Secondary Structure in Natively Unfolded Proteins: a-Synuclein. J. AM. CHEM. SOC 126, 2399-2408. (doi:10.1021/JA0356176)

40. Khosa MA, Wu J, Ullah A. 2013 Chemical modification, characterization, and application of chicken feathers as novel biosorbents. RSC Adv. 3, 20800. (doi:10.1039/c3ra43787f)

41. Church JS, Poole AJ, Woodhead AL. 2010 The Raman analysis of films cast from 
dissolved feather keratin. Vib. Spectrosc. 53, 107-111.

(doi:10.1016/j.vibspec.2010.02.011)

42. Jackson M, Mantsch H. 1995 The use and misuse of FTIR spectroscopy in the determination of protein structure. Crit. Rev. Biochem. Mol. Biol. 30, 95-120.

43. Chirgadze YN, Fedorov O V., Trushina NP. 1975 Estimation of amino acid residue side-chain absorption in the infrared spectra of protein solutions in heavy water. Biopolymers 14, 679-694. (doi:10.1002/bip.1975.360140402)

44. Almutawah A, Barker SA, Belton PS. 2007 Hydration of gluten: A dielectric, calorimetric, and fourier transform infrared study. Biomacromolecules (doi:10.1021/bm061206g)

45. Choe C, Schleusener J, Lademann J, Darvin ME. 2017 Keratin-water-NMF interaction as a three layer model in the human stratum corneum using in vivo confocal Raman microscopy. Sci. Rep. 7, 15900. (doi:10.1038/s41598-017-16202-x)

\section{Captions}

Figure 1: [Feather anatomy schematic]

(a) A diagram showing the major parts of the feather. The shaft in this diagram has a subcutaneous part called the calamus and an extracutaneous part called the rachis.

(b) This shows that an excised piece of shaft was embedded in epoxy resin and polished to prepare the sample for Raman spectra to be taken, from 8 locations in (c) shown by red lines. (c) This shows a cross-section of the shaft.

Two layers of the cortex were observed in samples cut at up to $~ 30 \%$ of shaft length. For samples cut at longer lengths, only one layer, corresponding to the thicker inner layer, observed at shorter lengths, was present.

Figure 2 [Example of a complete Raman spectrum]

Example spectrum from a sample from a Swan, cut at $40 \%$ shaft length. It is from the outer region of the dorsal position, of a feather (P4) from the left wing. The Amide I, Amide III and S-S regions have been assigned and fitted following the method outlined in the text and the Supplementary Information (see also Tables 2-4).

Figure 3: [Amide I Spectrum] An example fit of a spectrum in the Amide I region. The pink line is the recorded spectrum, six component Gaussian functions are shown in grey and the black line is sum of these six components. The spectrum is from the outer region of the dorsal part of the fourth primary feather from the left wing of a Mallard, taken at $40 \%$ length from the base of the shaft. For the fitted spectrum $\mathrm{R}^{2}=0.997$.

Figure 4: [Amide III Spectrum] An example fit of a spectrum in the Amide III region. The pink line is the recorded spectrum, six component Gaussian functions are shown in grey and the black line is sum of these six components. The spectrum is from the outer region of the dorsal part of the fourth primary feather from the left wing of a Mallard, taken at $40 \%$ length from the base of the shaft. For the fitted spectrum $R^{2}=0.977$.

Figure 5: [S-S Spectrum] An example fit of a spectrum in the SS region. The pink line is the recorded spectrum, three component Gaussian functions are shown in grey and the black line is sum of these three components. The spectrum is from the outer region of the dorsal part of 
the fourth primary feather from the left wing of a Mallard, taken at $40 \%$ length from the base of the shaft. For the fitted spectrum $\mathrm{R}^{2}=0.995$.

Figure 6: [Amide I band intensity ratios]

Band intensity ratios $1668 \mathrm{~cm}^{-1}: 1613 \mathrm{~cm}^{-1}$ in the Amide I region of feathers from a Gull, Kestrel, Mallard and Swan cut at $40 \%$ shaft length. These bands correspond to $\beta$-sheet and side-chain respectively. Values for feathers from the left $(\mathrm{L})$ and right $(\mathrm{R})$ wings of the same bird are shown, and the order is Inner L, then Inner R; Outer L, then Outer R. This is indicated in the graph by the characters ' $L$ ' and ' $R$ '

Figure 7 [Amide III band intensity ratios]

Band intensity ratios $1262 \mathrm{~cm}^{-1}: 1240 \mathrm{~cm}^{-1}$ in the Amide III region of feathers from a Gull, Kestrel, Mallard and Swan cut at 40\% shaft length. These bands correspond to random coil and $\beta$-sheet respectively. Values for feathers from the left $(\mathrm{L})$ and right $(\mathrm{R})$ wings of the same bird are shown, and the order is Inner L, then Inner R; Outer L, then Outer R. This is indicated in the graph by the characters ' $L$ ' and ' $R$ '

Figure 8: [Amide I band intensity ratios]

Band intensity ratios $1668 \mathrm{~cm}^{-1}: 1613 \mathrm{~cm}^{-1}$ in the Amide I region of feathers from a Swan. Spectra were taken from cross-sections of feathers at 20, 40, 60 and $80 \%$ length from the base of the shaft, nine feathers were investigated (see text) 